\title{
Time analysis of alveolar ridge preservation using a combination of mineralized bone-plug and dense-polytetrafluoroethylene membrane: A histomorphometric study
}

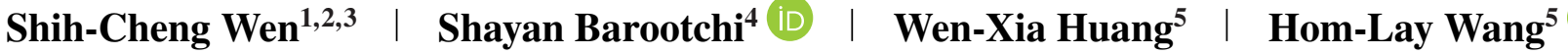

${ }^{1}$ Taipei Medical University, Taipei, Taiwan

${ }^{2}$ Private practice, Taipei, Taiwan

${ }^{3}$ Ching Kuo Institute of Management and Health, Keelung, Taiwan

${ }^{4}$ Department of Periodontics and Oral Medicine, School of Dentistry, University of Michigan, Ann Arbor, MI, USA

${ }^{5}$ Periodontics Department, Xiamen Stomatological Hospital, Xiamen, P. R. China

\section{Correspondence}

Dr. Hom-Lay Wang, DDS, MS, PhD, Department of Periodontics and Oral Medicine, University of Michigan School of Dentistry, 1011 North University Avenue, Ann Arbor, MI 48109-1078, USA.

Email: homlay@umich.edu

\begin{abstract}
Background: The objective of this study was to histologically evaluate and compare vital bone formation, residual graft particles, and fraction of connective tissue (CT)/other tissues between three different time points at 2-month intervals after alveolar ridge preservation with a cancellous allograft and dense-polytetrafluoroethylene (d-PTFE) membrane.
\end{abstract}

Methods: Ridge preservation with a cancellous allograft and d-PTFE membrane was performed at 49 extraction sockets (one per patient). Volunteers were assigned to implant placement at three different time points of 2, 4, and 6 months, at which time core biopsies were obtained. Histomorphometric analysis was performed to determine the percentages of vital bone, residual graft particles, and connective tissue/other nonbone components, and subjected to statistical analyses.

Results: There was a statistically significant difference in the amount of vital bone at every time point from $28.31 \%$ to $40.87 \%$ to $64.11 \%$ (at 2-, 4-, and 6-month groups, respectively) $(P<0.05)$. The percentage of residual graft particles ranged from $44.57 \%$ to $36.16 \%$ to $14.86 \%$, showing statistical significance from 4 to 6 months (21.29\%, $P<0.001)$, and 2 to 6 months $(29.71 \%, P<0.001)$, while there were no significant differences for the amount of CT/other tissue among the different time points.

Conclusions: This study provided the first histologic comparison of alveolar ridge preservation using a cancellous allograft and d-PTFE membrane at three different time points. Extraction sockets that healed for 6 months produced the highest amount of vital bone in combination with the least percentage of residual graft particles, while similar results were observed for the fraction of CT/other tissues between the three time points.

\section{K E Y W O R D S}

allografts, alveolar bone loss, alveolar resorption, bone transplantation, polytetrafluoroethylene, socket graft 


\section{1 | INTRODUCTION}

Extraction of unrestorable teeth and performing ridge preservation has become a routine clinical procedure in daily practices. The progressive resorption of the bony ridge that follows tooth extraction is a physiological phenomenon that can lead to potential esthetic and functional challenges for clinicians and patients. ${ }^{1,2}$ These changes in the alveolar dimensions can greatly alter treatment decisions and also interfere with ideal implant placement. ${ }^{3,4}$

To minimize this physiologic process, several therapeutic attempts have been investigated, a concept defined as "alveolar ridge preservation" (ARP). ${ }^{4}$ Over the past 2 decades, studies have used different techniques and materials, all aimed at reducing the post-extraction alveolar ridge atrophy. ${ }^{5-7}$ Among many different materials that have been used throughout the literature, the autogenous bone has been regarded as the gold standard for replacing or regenerating the resorbed alveolar ridge due to the fact that it consists of all necessary properties required in bone regeneration (osteoconduction, osteoinduction, and osteogenesis). ${ }^{8}$ However, the concerns of limited supply and donor site morbidity have urged many clinicians toward using the human allograft, which dominantly shares the second higher option for orthopedic surgeons and nearly one third of all bone grafts used in North America as they can be obtained in various forms and large quantities. ${ }^{9,10}$ The allogenic bone substitute has been extensively investigated for its properties to provide an adequate amount of vital bone for implant placement and its ability in reducing the resorption that occurs after tooth extraction. ${ }^{2,11-13}$ Another advantage of this material is its rapid turnover and replacement by the host bone. ${ }^{14,15}$ A high safety, sterile material, derived from human donor bone* been introduced that consists of a mineralized cancellous allograft, processed specifically for tissue preservation and viral inactivation. The properties of this bone substitute also include enhanced osteoconductivity, supporting natural and controlled tissue remodeling as well as preserved biomechanical characteristics.

Additionally, the use of a collagen wound dressing material has also been recommended for protection of the bone graft material as well as induction of blood clot formation and stabilization of the wound. ${ }^{16,17}$ The collagen dressing material is a homeostatic agent that possesses the ability to stimulate platelet aggregation and enhance fibrin linkage which may lead to initial clot formation, stability, and maturation. ${ }^{18,19}$ Additionally, collagen has shown to provide chemotactic properties for fibroblasts which could enhance cellular migration and promote wound closure, fundamental properties for bone growth and formation. ${ }^{20}$

\footnotetext{
* Botiss, Maxgraft cancellous, Zossen, Germany.
}

The use of barrier membranes, whether absorbable or non-resorbable, for bone and tissue regeneration has been well-documented. ${ }^{3,21}$ Despite recent studies reporting predictable outcomes when bioabsorbable membranes are left exposed over an extraction socket, ${ }^{22-24}$ traditionally, the use of absorbable and porous synthetic membranes required primary closure (tension-free in particular), which can increase the surgical complexity, and disrupts the natural soft tissue architecture by decreasing the amount of keratinized tissue, and reduce the depth of the vestibula as well. ${ }^{25,26}$

The dense-polytetrafluroethylene (d-PTFE) membrane was introduced for reducing bacterial contamination due to its high density and submicron pore size $(0.2 \mu \mathrm{m}) .{ }^{27,28}$ Additionally, it eliminates the need for obtaining a primary wound closure during the surgery and facilitates membrane removal at the postoperative visit. ${ }^{25,29}$ The efficacy of this barrier membrane has been demonstrated in preclinical and human studies with regard to augmenting an extraction sockets before implant placement. ${ }^{13,21,30-32}$

The ideal time of implant placement in an augmented ridge however is still a controversial topic for many clinicians and patients who are constantly at pursuit for expedited replacement therapy. ${ }^{33}$ Therefore, this study was designed to histologically compare the healing of augmented extraction sockets treated at 2, 4, and 6 months using a combination of mineralized bone allograft-plug socket augmentation ${ }^{18}$ with a d-PTFE membrane for differences in the amount of vital bone, residual graft particles, and connective tissue (CT)/other.

\section{2 | MATERIALS AND METHODS}

\section{1 | Study design and patient selection}

The present investigation was designed as a prospective controlled clinical study to evaluate the histologic healing of treated extraction socket at three different time points with equal time intervals. A total of 49 individuals (32 females, 17 males) were recruited and included within the study from a pool of patients at a private practice in Taipei, Taiwan. The following criteria were set for inclusion: (1) healthy adults in need of at least one tooth extraction either due to periodontal involvement, severe unrestorable decay, or failed endodontic therapy, (2) desiring restoration with a dental implant. Conversely, the criteria for exclusion were (1) sockets exhibiting severe loss of bony walls after extraction $(<50 \%$ dehiscence after tooth extraction), (2) a reported a smoking habit of $\geq 10$ cigarettes per day, (3) teeth with short or malpositioned roots such that the core biopsy might include bone along with the socket wall, (4) pregnancy, and (5) any medical conditions or medications known to alter soft tissue or bony healing (poorly controlled diabetes mellitus, bisphosphonates, immunosuppressives, etc.). 

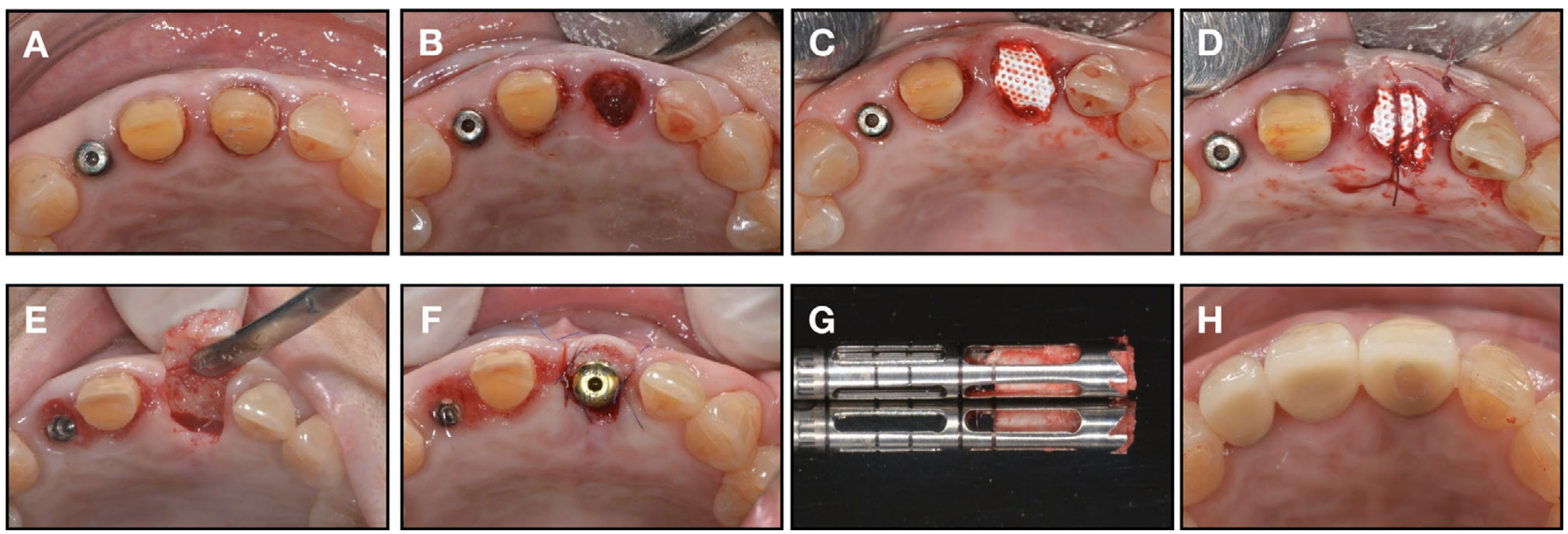

F I G U R E 1 An example of a case of an extraction of a hopeless tooth and alveolar ridge preservation with a cancellous allograft and d-PTFE: A) the hopeless tooth; B) view of the extraction socket; C) placement of a d-PTFE membrane after densely packing the socket with a cancellous allograft and placement of a collagen dressing; D) placement of cross sutures on top of the d-PTFE membrane and intentionally leaving it exposed; E) view of the augmented ridge after 6 months (in this case); F) placement of an appropriate size implant after G) obtaining a 2-mm bone core biopsy; and $\mathbf{H}$ ) final restoration of the placed implant

After successful fulfilling the criteria, the study protocol was thoroughly explained to eligible patients, at which point they were asked to complete a full medical history, provide informed consents, and a periapical radiograph was taken of the tooth that was scheduled for extraction. The study protocol, in full accordance with the Declaration of Helsinki of 1965, revised in Tokyo in 2004, was approved by the Institutional Review Board (Stomatological Hospital of Xiamen Medical College) and received the approval by the local ethics committee.

\section{2 | Surgical protocol}

No pre-surgical antibiotics were provided. All surgical procedures for the recruited individuals were performed by one experienced surgeon (SW) after the same measures and rationales of previously published protocols by the same research group ${ }^{17,18}$ (The Mineralized Bone Allograft-Plug Socket Augmentation Technique). Briefly, after successful administration of local anesthesia, a no. 15C scalpel blade was used for severing the supracrestal gingival fibers followed by using a long slender fine diamond bur to widen the periodontal ligament space. The tooth was then luxated with elevators and gently extracted with forceps. In case of a molar extraction, if necessary, the crowns were sectioned to remove the roots individually. The sockets were closely examined for the presence of a fenestration or dehiscence and thoroughly degranulated. A periodontal probe* was used to take measurements of the extraction socket on the facial and lingual/palatal sites.

The walls of the sockets were scraped with a curet and/or a no. 1/2 round bur to induce bleeding and then grafted and densely packed till $1-\mathrm{mm}$ below the bony crest with

\footnotetext{
*UNC-15 periodontal probe, Hu-Friedy, Chicago, IL.
}

a mineralized cancellous allograft.* Next a bioabsorbable collagen dressing ${ }^{\dagger}$ was gently packed on top of the bone graft for achieving stability. Last, a non-resorbable d-PTFE membrane ${ }^{\ddagger}$ was trimmed to the shape and size of the extraction socket and passively placed over the collagen dressing. Cross mattress sutures ${ }^{\S}$ were performed to secure and stabilize the d-PTFE membrane and approximate the tissues. At the end of the surgical procedure, a panoramic radiograph was taken. The membrane was left intentionally exposed until 4 weeks at which point the membrane along with the sutures were removed. Figure 1 is an example of a surgical procedure. All extractions were performed in a flapless manner. If $<50 \%$ of any socket wall was absent, the site was excluded from the analysis.

After the completion of the surgical procedure, the patients were assigned to the 2-, 4-, or 6-month groups at which point they would return for implant placement and bone core biopsies. The order of assignment was conducted in a sequential manner in which the first 19 individuals that were successfully enrolled in the study were allocated to the 6-month group, followed by the next 18 patients who were enrolled in the 4-month healing group, and the last 12 which were allocated to the 2-month healing group.

Postoperative instructions consisted of antibiotics (amoxicillin $500 \mathrm{mg}$ for 7 days, three times daily or in case of allergies 6 tablets of zithromax $250 \mathrm{mg}, 2$ tablets for the first day followed by one for the remaining 4 days) after the alveolar ridge preservation procedure, and anti-inflammatories as indicated. Patients were asked to rinse twice daily with warm salt

\footnotetext{
Collacone, Botiss, Zossen, Germany.

* Cytoplast TXT-200, Osteogenics Biomedical, Lubbock, TX.

$\S$ Cytoplast PTFE, Osteogenics Biomedical, Lubbock, TX.
} 
water for the first 2 weeks before switching to twice daily rinsing with $0.12 \%$ chlorhexidine gluconate mouthwash.

\section{3 | Re-entry procedure and implant placement}

At the appropriate time points, the patients returned for the second surgical visit and implant therapy. A mucoperiosteal flap was reflected and the initial clinical measurements were repeated, and a bone biopsy was harvested (according to the position of the future implant). A surgical trephine bur drill with an internal diameter of $2 \mathrm{~mm} *$ with depth indictor markings was used to obtain a core biopsy and the remaining osteotomy was completed according to the protocol of the manufacturer to receive an appropriate size, diameter, and length implant. ${ }^{\dagger}$ If necessary additional ridge augmentation at the time of implant placement was performed and recorded. The patients were seen 7 to 10 days postoperatively and the definitive restoration was inserted according to the treatment plan design within 12 months of the implant placement.

\section{4 | Histology processing}

The harvested bone cores were removed from trephines and placed directly into $10 \%$ neutral buffered formalin solution. Cores were decalcified, dehydrated, embedded in paraffin, and sectioned to 4- $\mu \mathrm{m}$-thick blocks for histomorphometric analysis. The slides were stained with H\&E and Masson trichrome and each section was examined at a low magnification prior to high power evaluation for determining the one which would provide the best section for the final assessment.

\section{5 | Histomorphometric analysis}

Following histology preparation, selected specimens were evaluated histomorphometrically using a Nikon Eclipse 50i microscope and a digital camera (at the same standardized magnification) to identify the following components: vital bone as the presence of osteocytes in lacunae, residual graft particles identified as regions of lamellar bone presenting lacunae without osteocytes, and CT/other which included vasculature, loose fibrous CT, and inflammatory cells. Once a core was separated into its respective regions (vital bone, residual graft particles, CT/other), each image was converted into a black and white photo, and the total number of pixels were calculated and summed for each image, and the percentage of the pixels was calculated to obtain the amount (in \%) of each component from the harvested bone core. All measurements and analyses were completed using a combination

\footnotetext{
* Salvin Dental Specialties, Charlotte, NC.

† Zimmer TSV, Zimmer/Biomet3i, West Palm Beach, FL.
}

of photo processing and conversion software ${ }^{\ddagger \|}$ and specified analysis software. ${ }^{\S}$

\subsection{Data and statistical analysis}

Means and standard deviations were calculated for the amount of vital bone (\%), residual graft particles (\%), and CT/other materials $(\%)$ at the three different time points $(2,4$, and 6 months). ANOVA was used for testing the presence of statistically significant differences between the three groups (2, 4 , and 6 months). A $P$ value threshold of 0.05 was set for statistical significance, at which case post-hoc analysis was performed to determine the source of variability. Additionally, box plots were produced to visualize the outcomes. All analyses were conducted in specified statistical software, ${ }^{\text {II }}$ and the plots were produced with the ggplot2 package. ${ }^{34}$

\section{3 | RESULTS}

All surgical procedures were successfully performed without complications. Each patient contributed to one treated extraction socket. Bone core biopsies were harvested from every augmented site and implants were placed for all patients at their specified time, followed by permanent restorations. Without occurrences of dropouts. The reasons for tooth extraction included: non-restorable dentition due to severe decay $(42.8 \%)$, or fracture (18.3\%), and periodontal disease (38.7\%).

Table 1 displays the patient characteristics and distribution of extraction sockets at baseline according to the assigned healing groups. Following extraction, no socket exhibited a dehiscence of $>50 \%$ of the buccal plate. The mean age of patients at the time of enrollment was $51.32 \pm 14.4$ years (54.8 for the 2-month group, 51.3 for the 4-month group, and 49.1 years for the 6-month group). Regarding the sex distribution, the percentage of males in the 2, 4, and 6-month groups was $33.3 \%, 22.2 \%$, and $47.36 \%$, respectively. Additionally, other patient characteristics were also not statistically different at baseline among the three groups. Fifty percent of the extracted teeth in the 2-month group, $61.1 \%$ in the 4month group, and $68.5 \%$ of the teeth in the 6-month group were molars.

\section{1 | Histology and histomorphometric findings}

Figure 2 displays the histomorphometric outcomes at three different time points stained with $\mathrm{H} \& \mathrm{E}$ and Masson

\footnotetext{
Adobe Photoshop CS6, Adobe Systems, San Jose, CA.

$\S$ ImageJ, National Institutes of Health, Bethesda, MD.

II Rstudio, Version 1.1.383, Rstudio, Boston, MA
} 
T A B L E 1 Characteristics of the included patients and treated sockets at baseline

\begin{tabular}{|c|c|c|}
\hline Group & Characteristic & Value \\
\hline \multicolumn{3}{|c|}{ 2-month healing group } \\
\hline & Participants (n) & 12 \\
\hline & Age, mean $\pm \mathrm{SD}$ & $54.8 \pm 15.4$ years \\
\hline & Males (n) & 4 \\
\hline & Maxillary sockets (n) & 1 \\
\hline & Mandibular sockets (n) & 11 \\
\hline & Incisors (n) & 0 \\
\hline & Premolar (n) & 6 \\
\hline & Molar (n) & 6 \\
\hline \multicolumn{3}{|c|}{ 4-month healing group } \\
\hline & Participants (n) & 18 \\
\hline & Age, mean \pm SD & $51.3 \pm 15.5$ years \\
\hline & Males (n) & 4 \\
\hline & Maxillary sockets (n) & 15 \\
\hline & Mandibular sockets (n) & 3 \\
\hline & Incisors (n) & 5 \\
\hline & Premolar (n) & 2 \\
\hline & Molar (n) & 11 \\
\hline \multicolumn{3}{|c|}{ 6-month healing group } \\
\hline & Participants (n) & 19 \\
\hline & Age, mean \pm SD & $49.1 \pm 13.3$ years \\
\hline & Males (n) & 9 \\
\hline & Maxillary sockets (n) & 11 \\
\hline & Mandibular sockets (n) & 8 \\
\hline & Incisors (n) & 1 \\
\hline & Premolar (n) & 5 \\
\hline & Molar (n) & 13 \\
\hline
\end{tabular}

$\mathrm{n}$, treated sample size trichrome. The amount of vital bone for the 2-, 4-, and 6month group was $28.31 \% \pm 4.85 \%, 40.87 \% \pm 10.04 \%$, and $64.11 \% \pm 14.83 \%$, respectively $(P<0.001)$. Post-hoc pairwise comparisons revealed statistically significant differences between all the time points; from 2 to 4 months at $12.56 \%$ $(P=0.01)$, from 4 to 6 months at $23.23 \%(P<0.001)$, and from 2 to 6 months at $35.79 \%(P<0.001)$. The percentage of residual graft particles in the harvested bone biopsies ranged from $44.57 \% \pm 10.63 \%$ for the 2-month group, to $36.16 \% \pm$ $14.82 \%$ in the 4-month group, and $14.86 \% \pm 7.99 \%$ for the 6-month group. The overall comparison reached statistical significance $(P<0.001)$, and pair-wise comparisons showed statistically significant differences from 4 to 6 months (21.29\%, $P<0.001)$, and 2 to 6 months $(29.71 \%, P<0.001)$, while the difference from 2 to 4 months lacked statistical significance $(8.41 \%, P=0.14)$. With regard to fraction of CT/other, there was no significant differences among the different time points (2-month group: $27.12 \% \pm 10.6 \%$, 4-month group: $22.97 \% \pm 10.7 \%, 6$-month group: $22.08 \% \pm 10.9 \%$, $P=0.43$ ) (Fig. 3).

\section{4 | DISCUSSION}

Many studies have evaluated the success of ARP involving different type of bone grafts and barrier membranes. ${ }^{3,21,29,30}$ However, only some studies have reported on the healing of treated extraction sockets at different time points. In the present study, we sequentially evaluated the healing of sockets augmented with a cancellous allograft and a d-PTFE membrane at 2, 4, and 6 months to compare the amount of vital bone, residual graft particles, and CT/other tissues.

The importance of vital bone on implant success and survival is still unclear at this point. Regardless, it is reasonable to assume that a higher amount of vital bone is desirable for

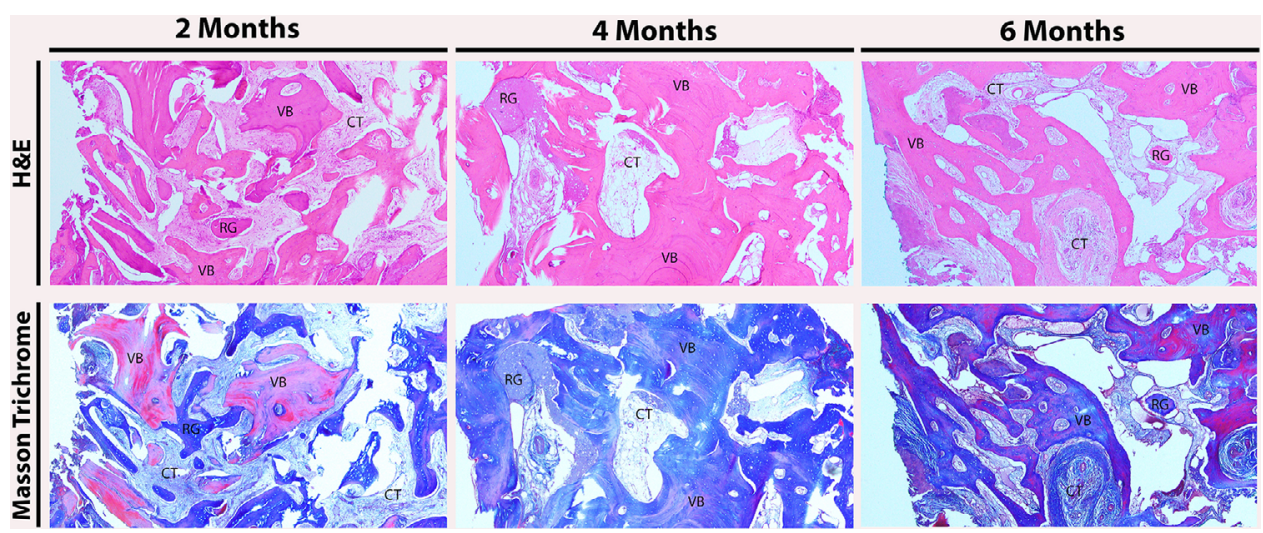

F I G U R E 2 Histology slides stained with H\&E and Masson trichrome from the cores obtained at 2, 4, and 6 months of augmented sockets with cancellous allograft and d-PTFE, showing new vital bone formation, remaining graft particles, and CT/other tissues. VB = vital bone (with osteocytes present in lacunae); $\mathrm{RG}=$ residual graft particles (with empty lacunae); $\mathrm{CT}=$ non-mineralized connective tissue/other material. (original magnification: $\times 100$ ) 


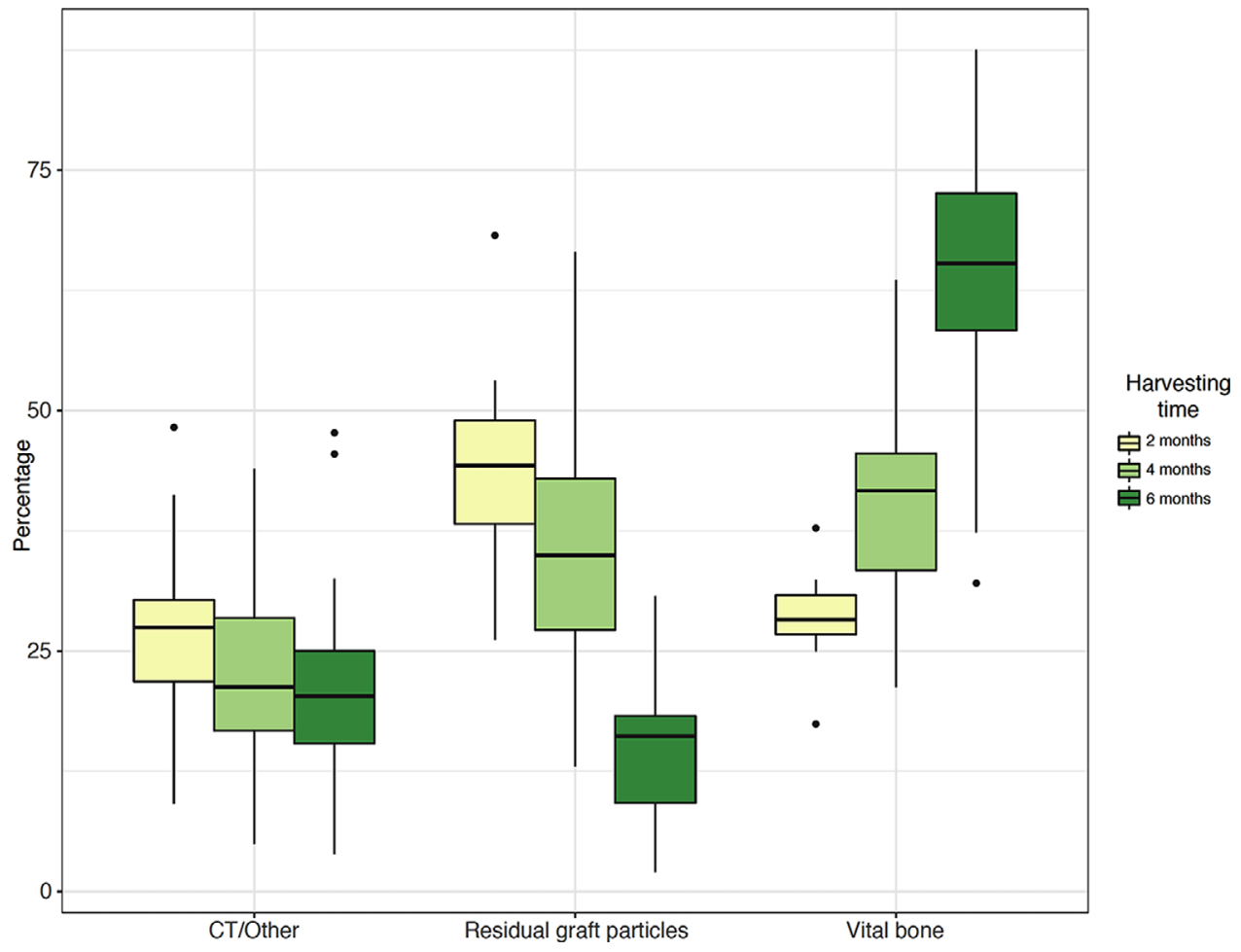

F I G U R E 3 Box plots showing the comparison of the histomorphometric outcomes in terms of percentage of CT/other tissue, residual graft particles, and vital bone formation, at 2, 4, and 6 months

increasing the bone-to-implant contact and accelerating the point at which implants can achieve stability.

Our histomorphometric findings demonstrated a significant increase in the percentage of vital bone at every evaluated time point ( $28 \%$ versus $41 \%$ versus $64 \%$ ), and a significant reduction in the amount of residual graft particles from 4 to 6 months (36\% to 15\%). While no significant changes in the fraction of CT/other tissues throughout the 2- to 6-month time periods, and almost no changes from 4 to 6 months $(27 \%, 23 \%$, and $22 \%$ ) were observed. To our best knowledge, a comparison of three different time points and with 2-month intervals has not been performed yet, and therefore, an exact approximation of our results to the literature may not be feasible.

Beck and Mealey were the first to examine the differences in new bone formation at two varying time intervals after ARP using the same human mineralized allograft. The authors found no significant differences in vital bone $(45 \%$ versus $46 \%$ ), residual graft particles (14\% versus $15 \%$ ), and CT/other ( $40 \%$ versus $41 \%$ ) between sites that had healed for an average of 3 months compared with those that had healed for an average of 6 months. ${ }^{35}$

The same research group later evaluated the healing of extraction sockets that were augmented with demineralized freeze-dried allograft (DFDBA) at $\approx 2$ to 2.5 months (shortterm) versus 4.5 to 5 months (long-term). ${ }^{11}$ Their results indicated a significantly greater vital bone formation for their long-term healing group (32.63\% versus $47.41 \%$ ), and despite a trend for decreased residual graft particles in the long-term group (37.42\% versus $26.8 \%$ ), they observed no differences for the fraction of CT/other tissues (29.94\% versus $25.78 \%$ ). In comparison with their former study, the authors speculated that the demineralization process of DFDBA which contributes to the release of bone morphogenetic proteins and benefits the allograft with osteoinductivity may have been an important factor, leading to the superior vital bone percentages at the corresponding time points.

The findings of our present study at 4 months are similar to that reported by Whetman and Mealey ${ }^{11}$ in their long-term group for the amount of vital bone formation (41\% versus $47 \%$, respectively). However, it should be noted that both mentioned studies used an absorbable collagen membrane in contrast to our d-PTFE membrane for covering the extraction socket.

Fotek et al. ${ }^{13}$ studied the effect of ARP with mineralized cancellous allograft comparing d-PTFE versus acellular dermal matrix membranes. Their histology results for both groups at 4 months are comparable with our 4-month data in terms of vital bone percentages $(41.8 \%$ for ADM, $47.36 \%$ for d-PTFE), while they found a significantly less amount of residual graft particles in both groups (13.93\% for ADM, $14.73 \%$ for d-PTFE).

The advantages of using a d-PTFE membrane include the possibility of leaving the membranes intentionally exposed without compromising the healing process, preserving 
the alveolar bone, and the likelihood of increasing the amount of keratinized mucosa. ${ }^{27,28}$ The efficacy of this barrier membrane with other graft materials has also been demonstrated. ${ }^{13,21,30-32}$

The current study is not without potential confounding variables. The source of our bone graft material was not from a single donor. This could lead to some variability in the residual calcium content, as well as the inclusion of both single and multi-rooted teeth may have had some influence on the outcomes. Additionally, no attempt was made to correlate the histomorphometric results to the dimensional changes due to the lack of a standardized custom stent for the ridge measurements, and last, the lack of a control group in the current study, wherein sockets heal spontaneously without any intervention may introduce a notable limitation when comparing the histologic results of the augmented sites. Thus, care should be taken in regard to the conclusions and generalizability of our results.

\section{5 | CONCLUSIONS}

Within the scope of the current investigation, it can be concluded that after tooth extraction and ridge preservation with mineralized cancellous allograft and d-PTFE membrane, a significantly greater amount of vital bone formation occurs with a reduction in residual graft particles when comparing 2 to 4 to 6 months after socket grafting.

\section{ACKNOWLEDGMENTS}

The authors do not have any financial interests, either directly or indirectly, in the products or information listed in the paper except Dr. Hom-Lay Wang, who has occasionally received honoraria for speaking on behalf of Osteogenics Biomedical (Lubbock, TX). This paper was partially supported by the University of Michigan Periodontal Graduate Student Research Fund.

\section{ORCID}

Shayan Barootchi (D) https://orcid.org/0000-0002-5347-6577 Hom-Lay Wang (ID) https://orcid.org/0000-0003-4238-1799

\section{REFERENCES}

1. Amler MH, Johnson PL, Salman I. Histological and histochemical investigation of human alveolar socket healing in undisturbed extraction wounds. J Am Dent Assoc. 1960;61:32-44.

2. Iasella JM, Greenwell H, Miller RL, et al. Ridge preservation with freeze-dried bone allograft and a collagen membrane compared to extraction alone for implant site development: a clinical and histologic study in humans. J Periodontol. 2003;74:990-999.

3. Lekovic V, Camargo PM, Klokkevold PR, et al. Preservation of alveolar bone in extraction sockets using bioabsorbable membranes. J Periodontol. 1998;69:1044-1049.

4. Darby I, Chen S, De Poi R. Ridge preservation: what is it and when should it be considered. Aust Dent J. 2008;53:11-21.

5. Aimetti M, Romano F, Griga FB, Godio L. Clinical and histologic healing of human extraction sockets filled with calcium sulfate. Int J Oral Maxillofac Implants. 2009;24:902-909.

6. Barone A, Aldini NN, Fini M, Giardino R, Calvo Guirado JL, Covani $\mathrm{U}$. Xenograft versus extraction alone for ridge preservation after tooth removal: a clinical and histomorphometric study. J Periodontol. 2008;79:1370-1377.

7. Barone A, Toti P, Quaranta A, et al. Clinical and histological changes after ridge preservation with two xenografts: preliminary results from a multicentre randomized controlled clinical trial. J Clin Periodontol. 2017;44:204-214.

8. Bauer TW, Muschler GF. Bone graft materials. An overview of the basic science. Clin Orthop Relat Res. 2000:10-27.

9. De Long WG, Jr, Einhorn TA, Koval K, et al. Bone grafts and bone graft substitutes in orthopaedic trauma surgery. A critical analysis. J Bone Joint Surg Am. 2007;89:649-658.

10. Fillingham Y, Jacobs J. Bone grafts and their substitutes. Bone Joint J. 2016;98-B:6-9.

11. Whetman J, Mealey BL. Effect of healing time on new bone formation after tooth extraction and ridge preservation with demineralized freeze-dried bone allograft: a randomized controlled clinical trial. J Periodontol. 2016;87:1022-1029.

12. Wood RA, Mealey BL. Histologic comparison of healing after tooth extraction with ridge preservation using mineralized versus demineralized freeze-dried bone allograft. J Periodontol. 2012;83:329336

13. Fotek PD, Neiva RF, Wang HL. Comparison of dermal matrix and polytetrafluoroethylene membrane for socket bone augmentation: a clinical and histologic study. J Periodontol. 2009;80:776-785.

14. Sheikh Z, Hamdan N, Ikeda Y, Grynpas M, Ganss B, Glogauer M. Natural graft tissues and synthetic biomaterials for periodontal and alveolar bone reconstructive applications: a review. Biomater Res. 2017;21:9.

15. Wang W, Yeung KWK. Bone grafts and biomaterials substitutes for bone defect repair: a review. Bioact Mater. 2017;2:224-247.

16. Sclar AG. Preserving alveolar ridge anatomy following tooth removal in conjunction with immediate implant placement. The Bio-Col technique. Atlas Oral Maxillofac Surg Clin North Am. 1999;7:39-59.

17. Wang HL, Kiyonobu K, Neiva RF. Socket augmentation: rationale and technique. Implant Dent. 2004;13:286-296.

18. Wang HL, Tsao YP. Mineralized bone allograft-plug socket augmentation: rationale and technique. Implant Dent. 2007;16:33-41.

19. Sabelmann E. Biology, biotechnology, and biocompatibility of collagen. In Williams DF, ed. Biocompatibility of Tissue Analogues. CRC Press; 1985:27-66.

20. Postlethwaite AE, Seyer JM, Kang AH. Chemotactic attraction of human fibroblasts to type I, II, and III collagens and collagenderived peptides. Proc Natl Acad Sci U S A. 1978;75:871-875.

21. Laurito D, Lollobrigida M, Gianno F, Bosco S, Lamazza L, De Biase A. Alveolar ridge preservation with nc-HA and d-PTFE membrane: a clinical, histologic, and histomorphometric study. Int $J$ Periodontics Restorative Dent. 2017;37:283-290. 
22. Barone A, Toti P, Piattelli A, Iezzi G, Derchi G, Covani U. Extraction socket healing in humans after ridge preservation techniques: comparison between flapless and flapped procedures in a randomized clinical trial. J Periodontol. 2014;85:14-23.

23. Lee J, Lee JB, Koo KT, Seol YJ, Lee YM. Flap management in alveolar ridge preservation: a systematic review and meta-analysis. Int J Oral Maxillofac Implants. 2018;33:613-621.

24. Lee JS, Cha JK, Kim CS. Alveolar ridge regeneration of damaged extraction sockets using deproteinized porcine versus bovine bone minerals: a randomized clinical trial. Clin Implant Dent Relat Res. 2018;20:729-737.

25. Bartee BK. Evaluation of a new polytetrafluoroethylene guided tissue regeneration membrane in healing extraction sites. Compend Contin Educ Dent. 1998;19:1262-1254.

26. Wang HL, Carroll MJ. Guided bone regeneration using bone grafts and collagen membranes. Quintessence Int. 2001;32:504-515.

27. Carbonell JM, Martin IS, Santos A, Pujol A, Sanz-Moliner JD, Nart J. High-density polytetrafluoroethylene membranes in guided bone and tissue regeneration procedures: a literature review. Int J Oral Maxillofac Surg. 2014;43:75-84.

28. Krauser JT. High-density PTFE membranes: uses with root-form implants. Dent Implantol Update. 1996;7:65-69.

29. Barber HD, Lignelli J, Smith BM, Bartee BK. Using a dense PTFE membrane without primary closure to achieve bone and tissue regeneration. J Oral Maxillofac Surg. 2007;65:748-752.

30. Laurito D, Cugnetto R, Lollobrigida M, et al. Socket preservation with d-PTFE membrane: histologic analysis of the newly formed matrix at membrane removal. Int J Periodontics Restorative Dent. 2016;36:877-883
31. Bartee BK, Carr JA. Evaluation of a high-density polytetrafluoroethylene (n-PTFE) membrane as a barrier material to facilitate guided bone regeneration in the rat mandible. J Oral Implantol. 1995;21:88-95.

32. Walters SP, Greenwell H, Hill M, Drisko C, Pickman K, Scheetz JP. Comparison of porous and non-porous teflon membranes plus a xenograft in the treatment of vertical osseous defects: a clinical reentry study. J Periodontol. 2003;74:1161-1168.

33. De Risi V, Clementini M, Vittorini G, Mannocci A, De Sanctis M. Alveolar ridge preservation techniques: a systematic review and meta-analysis of histological and histomorphometrical data. Clin Oral Implants Res. 2015;26:50-68.

34. Wickham H. ggplot2: Elegant Graphics for Data Analysis. New York: Springer-Verlag; 2009.

35. Beck TM, Mealey BL. Histologic analysis of healing after tooth extraction with ridge preservation using mineralized human bone allograft. J Periodontol. 2010;81:1765-1772.

How to cite this article: Wen SC, Barootchi S, Huang WX, Wang HL. Time analysis of alveolar ridge preservation using a combination of mineralized bone-plug and dense-polytetrafluoroethylene membrane: A histomorphometric study. J Periodontol. 2020;91:215-222. https://doi.org/10.1002/JPER. 19-0142 